\title{
MICROWAVE AND MILLIMETER WAVE IMAGING OF THE SPACE SHUTTLE EXTERNAL FUEL TANK SPRAY ON FOAM INSULATION (SOFI) USING SYNTHETIC APERTURE FOCUSING TECHNIQUES (SAFT)
}

\author{
J.T. Case' ${ }^{\text {, J. Robbins }}{ }^{1}$, S. Kharkivskiy', F. Hepburn ${ }^{2}$ and R. Zoughi' \\ 'Applied Microwave Nondestructive Testing Laboratory (amntl), Electrical and Computer \\ Engineering Department, University of Missouri-Rolla, Rolla, MO 65409, USA \\ ${ }^{2}$ NASA George C. Marshall Space Flight Center, Huntsville, AL 35812, USA
}

\begin{abstract}
The Space Shuttle Columbia's catastrophic failure is thought to have been caused by a dislodged piece of external tank spray on foam insulation (SOFI) striking the left wing of the orbiter causing significant damage to some of the reinforced carbon/carbon leading edge wing panels. Microwave and millimeter wave nondestructive evaluation methods have shown great potential for inspecting SOFI for the purpose of detecting anomalies such as small air voids that may cause separation of the SOFI from the external tank during a launch. These methods are capable of producing relatively high-resolution images of the interior of SOFI particularly when advanced imaging algorithms are incorporated into the overall system. To this end, synthetic aperture focusing techniques (SAFT) are being developed. This paper presents some of the preliminary results of this investigation using SAFT-based methods and microwave holography at relatively low frequencies illustrating their potential capabilities for operation at millimeter wave frequencies.
\end{abstract}

Keywords: microwave holography, SAFT, SOFI

PACS: 42.30.Va, 42.40.Jv, 84.40.-x

\section{INTRODUCTION}

Spray on foam insulation (SOFI) is used to insulate the external fuselage of the Space Shuttle to maintain fuel, liquid hydrogen and liquid oxygen, at cryogenic temperatures [1]. During a launch, pieces of the SOFI may break off ranging in size from a few cubic millimeters to thousands of cubic centimeters. One such piece is thought to have damaged the reinforced carbon/carbon panels on the wing of the obiter Columbia [1]. The presence of an interior small void (or debond) in SOFI provides for higher internal pressure in the void during the high speed launch and may cause surrounding SOFI to break and dislodge from the tank. Therefore, a robust nondestructive testing and evaluation (NDT\&E) method is desired that is capable of detecting small voids in SOFI prior to a launch. 
Microwave and millimeter wave NDT\&E methods have shown great potential to produce relatively high-resolution images of voids inside SOFI test panels $[2,3]$. These methods are expected to be even more effective when combined with advanced imaging algorithms. Two such methods are presented in this paper, namely; synthetic aperture focusing technique (SAFT) [4] and microwave holography [5]. These methods are in many ways similar to one another. For processing purposes, it is assumed that the wave is not delayed as it propagates through SOFI. This is valid because SOFI has a permittivity of only 1.05 at X-band (8.2-12.4 $\mathrm{GHz}$ ) as compared to air with a relative permittivity of $1.00[3,6]$. Therefore, image processing is used to aid in finding weak reflections occurring at air and SOFI interfaces (i.e., void boundaries). The essence of each technique is to match the phase of the wave signal originating an arbitrary located target to measured points spaced regularly on a plane. For a signal originating at that target, processed signals add constructively and they add destructively for all surrounding points, consequently providing significant spatial discrimination resulting in a high-resolution image.

Synthetic aperture focusing technique (SAFT) can be used to synthetically focus a single frequency microwave image to a specific focal plane. For this method, a large, narrow beam antenna is synthesized by measuring with a small, broad-beam antenna at regular points on a grid, which results in images with high spatial resolution. The SAFT method described and used here was derived in the frequency domain and utilizes angular spectrum decomposition [4]. The original microwave image is decomposed onto a basis set of plane waves emitted from the center of the synthetic antenna array using the 2D FFT. The waves are then back-propagated to the focal plane and then to the measurement plane accounting for roundtrip back-propagation, and then they are composed back into normal space using the inverse 2D FFT. This yields a high resolution 2D image with lateral resolution of one-half the length of the dimension of the actual small microwave antenna and is independent of range or distance to the target [7]. To augment range resolution and image quality, it is possible to perform this algorithm at many frequencies within a waveguide band and average the intermediate data before the inverse 2D FFT is performed. However, this method may not produce high quality images for an object greater than the depth of focus [8].

Microwave holography, on the other hand, can be used to generate a full 3D representation of an object or embedded defect [5]. Thus, the 3D position and shape of the defect may be determined. This method utilizes a swept frequency measurement over the measurement plane resulting in a dataset of $(x, y, f)$. During image processing, spatial dimensions $(x, y)$ are transformed to spatial frequency dimensions $\left(k_{x}, k_{y}\right)$ by operating the 2D FFT independently on every measured frequency, $f$. Frequency $f$ can then be related to spatial frequency $k_{z}$ using the following dispersion relation [5]

$$
k_{x}^{2}+k_{y}^{2}+k_{z}^{2}=(2 k)^{2}
$$

such that

$$
k=\frac{2 \pi f}{c}
$$

where $c$ is the speed of light in vacuum. Real datasets must be re-sampled onto a regular spacing of $k_{z}$. The dataset can then be transformed to $(x, y, z)$ from $\left(k_{x}, k_{y}, k_{z}\right)$ using the inverse $3 D$ FFT. Consequently, the resulting dataset is a volumetric representation of the defect and can 
be graphically illustrated as such. The spatial resolution is comparable to SAFT such that the range of the target is less than the dimension of the array. The range resolution associated with this holographic method is $c / 2 B$, where $B$ is the transmitted signal bandwidth [5]. For this paper, slices of the volume dataset are provided rather than a full three dimensional representation due to the nature of the samples used.

It is very important to note that this investigation uses relatively low frequencies that are not conducive for direct imaging of the SOFI $[2,3]$. However, as will be seen later, when using the image processing algorithms outlined above, reasonably high resolution images are obtained even at these relatively low frequencies. The ultimate goal of this investigation is to demonstrate the capability of these methods before they are applied to much higher frequencies like the millimeter wave frequency spectrum (e.g., 30-300 GHz).

\section{EXPERIMENTAL APPROACH}

All measurements were taken using an automated 2D scanner such that all measurement points were constrained to one plane. At each point, the microwave reflection coefficient at the aperture of an open-ended rectangular waveguide was measured. Three separate experiments were conducted for this investigation: inverse foam, void in foam, and foam with rind. The first experiment, inverse foam, utilizes SOFI in air, representing the inverse of a cube of void in SOFI. The inverse foam case was used because at the time there was no representative SOFI sample available. Thus, two SOFI cubes with side dimensions of $12.7 \mathrm{~mm}(0.5 \mathrm{in})$ were prepared and placed on an aluminum plate (substrate) $12.7 \mathrm{~mm}$ apart and $100 \mathrm{~mm}$ below the measurement plane. Measurements were taken at $\mathrm{Ku}$-band $(12.4-18 \mathrm{GHz})$ at a sampling increment of $5 \mathrm{~mm}$ using an HP8510C Vector Network Analyzer. The second experiment, void in foam, was conducted on a $70 \mathrm{~mm}$ thick SOFI slab with $25 \mathrm{~mm}$ (1 in) diameter flat bottom holes drilled at the following depths: $25 \mathrm{~mm}(1 \mathrm{in}), 19 \mathrm{~mm}(3 / 4 \mathrm{in}), 13 \mathrm{~mm}(1 / 2 \mathrm{in}), 6 \mathrm{~mm}(1 / 4$ in), and 3 (1/8 in). This slab was arranged in three different ways for three cases: 1) backed by substrate, 2) backed by another $70 \mathrm{~mm}$ thick SOFI slab with no holes (blank sample) and substrate, and 3) covered by $70 \mathrm{~mm}$ thick blank SOFI and backed by substrate. In this way the locations of the holes within the overall SOFI thickness varied. Liftoff, the distance between the probe and the SOFI surface, was $10 \mathrm{~mm}$. Measurements were taken at $\mathrm{K}$-Band (18-26.5 $\mathrm{GHz}$ ) at sampling increments of $2 \mathrm{~mm}$ using an Agilent $8361 \mathrm{~A}$ PNA Series Vector Network Analyzer. The third case, foam with rind, consisted of a large $560 \mathrm{~mm} \times 560 \mathrm{~mm} \mathrm{x} 76 \mathrm{~mm}(22$ in $x 22$ in $x 3$ in) SOFI sample with exposed rind backed by substrate. Measurements were taken at K-Band, $18-26.4 \mathrm{GHz}$, at a sampling increment of $2 \mathrm{~mm}$ and a liftoff of $10 \mathrm{~mm}$ using an Agilent 8361A PNA Series Vector Network Analyzer.

\section{RESULTS}

\section{Inverse Foam}

The first experiment analyzed is the inverse foam case of two SOFI cubes on top of a substrate. Using Friis transmission equation the reflection from the SOFI cubes was estimated to be $-70 \mathrm{~dB}$ in relation to the microwave power at the aperture of the waveguide. Using image theory the reflection from the substrate would be much greater at $-45 \mathrm{~dB}$ [9]. Although the relative signal strength from the SOFI cube is small, it is still above noise and within the measurement dynamic range of the network analyzer. The waveguide-to-air boundary at the opening of the waveguide aperture causes a large reflection and is the dominant contribution to the net reflection coefficient. However, contributions to the measured reflection coefficient by 
the waveguide-to-air boundary and the substrate can be coherently subtracted before image processing is implemented (i.e., calibration). This is done by subtracting the average reflection coefficient over the whole scan area, which is a reasonable estimation of both reflections since they are expected to remain constant over the scan area. Figure la shows the original image of the magnitude of reflection coefficient as measured by scanning the waveguide probe over the region of interest, which gives only a faint indication that something is present, and this is expected in a direct measurement before processing. Clearly, in this image there is no indication of two separate pieces of SOFI adjacent to one another. Figure $1 \mathrm{~b}$ shows the processed image using the SAFT algorithm described earlier. It is important to note that the contributions from the SOFI cubes, although small, can be resolved from the darker surroundings. It is also important to note that each SOFI cube can be resolved individually from the other even though the wavelength considered at $12.4 \mathrm{GHz}$ is $24 \mathrm{~mm}$ long compared to the size of the SOFI cubes, $12.7 \mathrm{~mm}$. This example clearly shows the effectiveness of this SAFT approach for obtaining high-resolution images. Figure 1c shows that microwave holography can further increase the quality of the image using the entire bandwidth available (12.4-18 $\mathrm{GHz}$ ). The quality is improved by further resolving the SOFI cubes and suppressing the background (or clutter) signal. This representative experiment demonstrates that the concept of using SAFT-based algorithms is not only practical, but also realizable despite the small waveguide probe power, small reflections from the SOFI, and comparatively large reflections from the substrate.

(a)

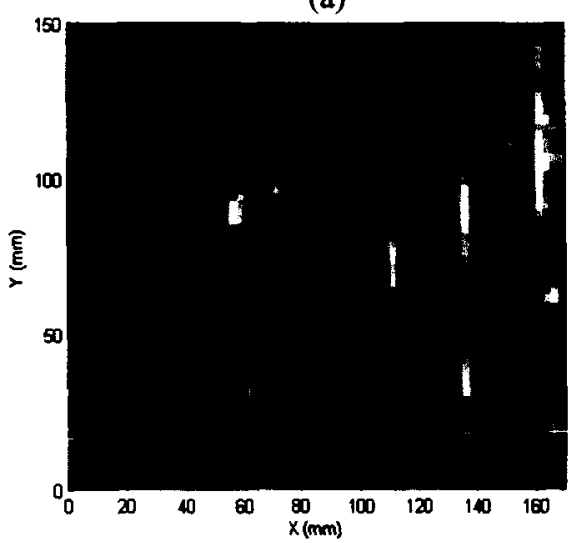

(b)

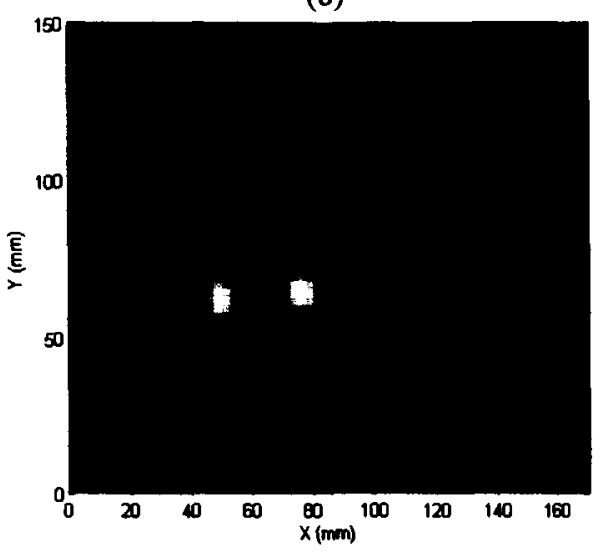

(c)

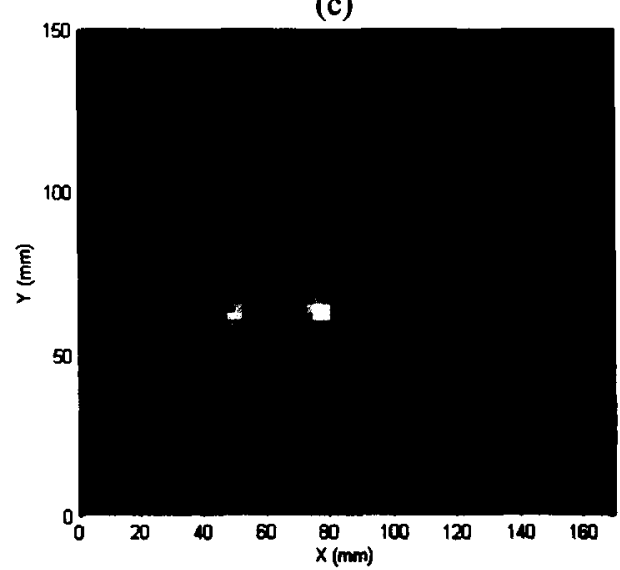

FIGURE 1. Inverse foam case: (a) original magnitude of reflection at $12.4 \mathrm{GHz}$, (b) SAFT processing at 12.4 $\mathrm{GHz}$ focusing at $105 \mathrm{~mm}$ from measurement plane, (c) microwave holography 
(a)

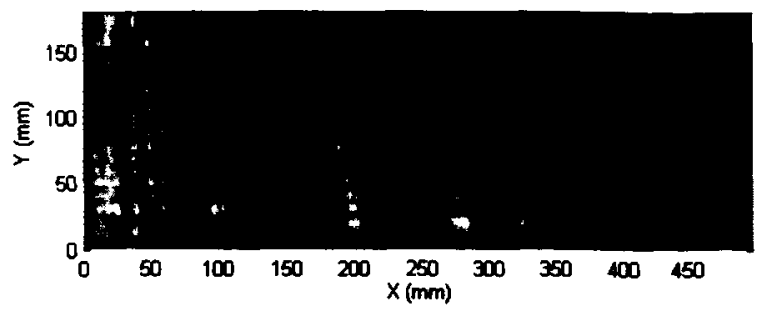

(c)

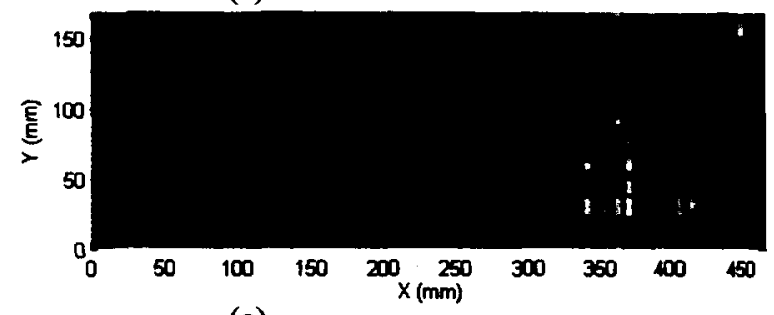

(e)

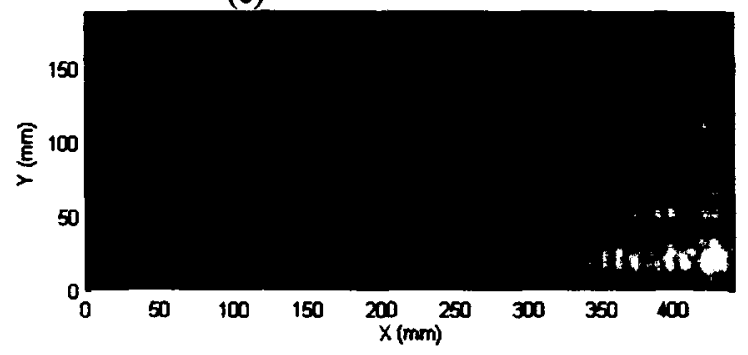

(b)

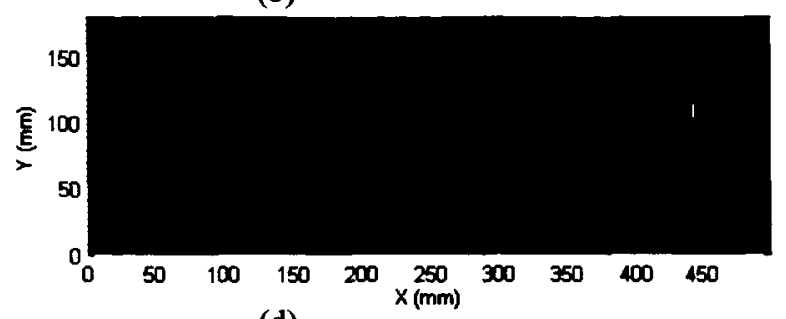

(d)

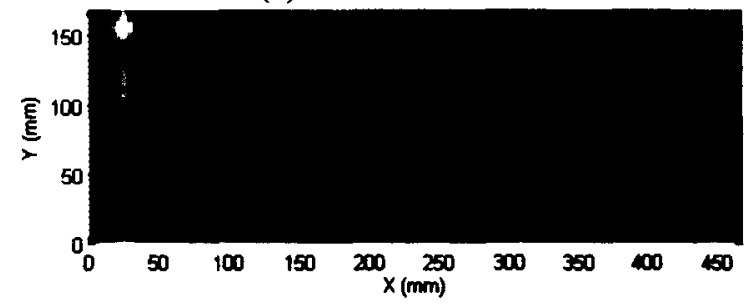

(f)

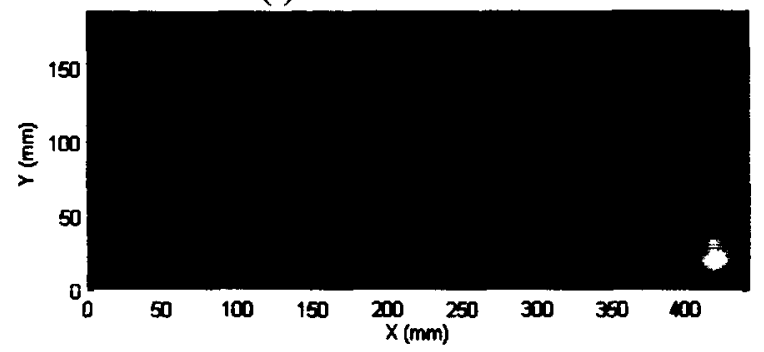

FIGURE 2. Void in foam: (a) case 1: SAFT processing at $22.25 \mathrm{GHz}$ focusing at $74 \mathrm{~mm}$ from measurement plane; (b) case 1: microwave holography slice at $74 \mathrm{~mm}(18-26.5 \mathrm{GHz})$; (c) case 2: SAFT processing at $22.25 \mathrm{GHz}$ focusing at 152 $\mathrm{mm}$; (d) case 2: microwave holography slice at $152 \mathrm{~mm}$; (e) case 3: SAFT processing at $22.25 \mathrm{GHz}$ focusing at $152 \mathrm{~mm}$; (f) case 3: microwave holography slice at $152 \mathrm{~mm}$.

\section{Void in Foam}

The first experiment demonstrated that foam could be detected in air. This experiment goes further to demonstrate the effectiveness of these algorithms for detecting voids in SOFI at different depths. This experiment was performed for three different cases as described in the experimental approach section. The first case consists of one SOFI slab with five flat bottom holes of different depths backed by an aluminum substrate. It is important to note the horizontal and vertical line aberrations occurring near the edges of the processed images. These are the result of two causes: the effects from the edges of the substrate and the rectangular, not square, nature of the dataset. Both can be minimized if the data set is cropped about a square region of interest, and future versions of the image processing code will minimize these aberrations. In Figure 2a, SAFT processing shows four of the five holes starting at the deepest on the right and descending towards the left. Microwave holography in Figure $2 \mathrm{~b}$ also shows four holes. The fifth hole is not evident from Figure $2 b$ since it is nearly masked by the edge effects. In spite of this, the fifth hole can be seen as a persistent indication when viewing the adjacent focal planes at different depths (not shown here).

The second case is for two SOFI slabs such that the slab containing the holes is nearest to the measurement plane and is backed by another slab and the aluminum substrate. The best images were obtained by focusing on the shadows of the voids cast onto the substrate. As seen in Figure 2c, SAFT processing is inadequate for such a thick sample and random noise seems 
to overwhelm the results except for a hint of the dominant hole on the far right. It is thought that the reduction in the quality of the image is due to low signal strength and low range resolution. Range resolution is dependent on the bandwidth of the system, but SAFT is inherently single frequency and as such provides low range resolution. Although range resolution is not usually necessary for a quality $2 \mathrm{D}$ image, the features sought for at a specific focal plane are blurred out along the direction of range. Therefore, the voids are effectively masked by the noise/clutter. Microwave holography uses the full available frequency spectrum and provides better results, as shown in Figure 2d, which shows four of the five holes for this case.

The third case is of two SOFI slabs where the slab with holes was sandwiched between another slab and the aluminum substrate. Once again, SAFT algorithm performed poorly due to reasons already described, as shown Figure 2e. However, four of the five holes were successfully detected using microwave holography. The last and smallest hole is again dominated by edge effects, as shown in Figure $2 \mathrm{f}$. It is important to note that this third case demonstrates that voids can be seen through $140 \mathrm{~mm}$ (5.5 in) of SOFI using this microwave holography.

(a)

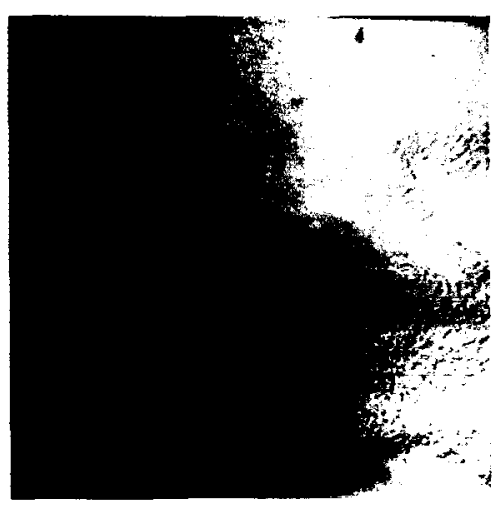

(c)

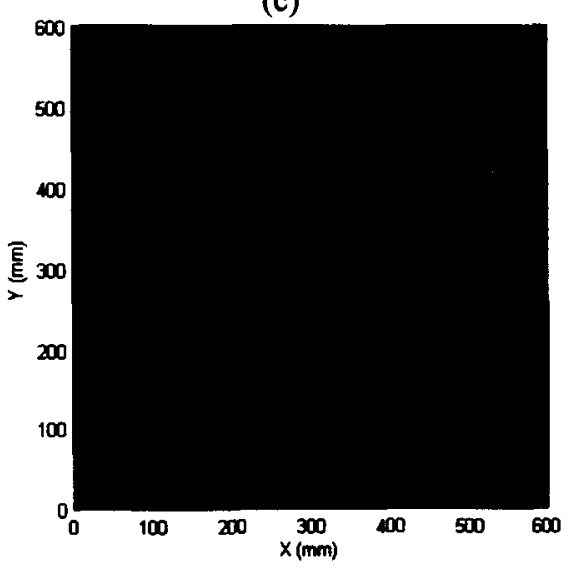

(b)

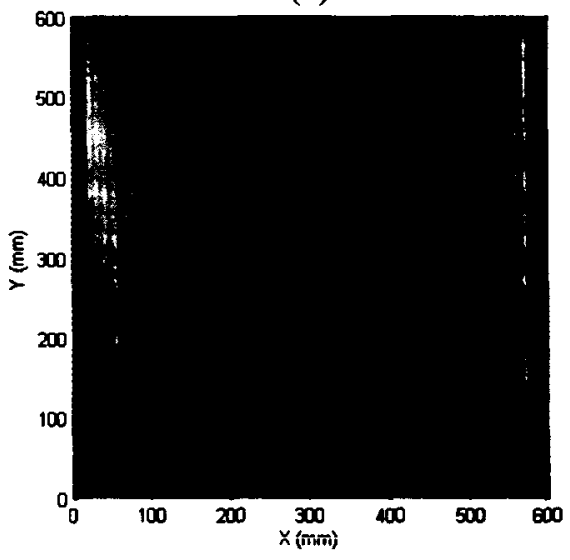

(d)

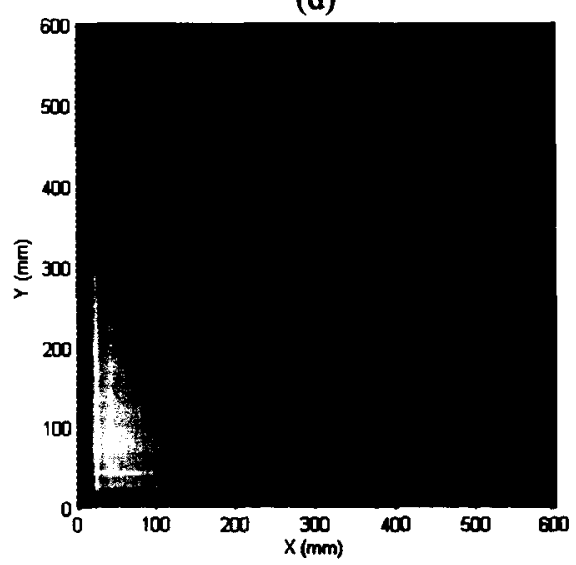

FIGURE 3. Foam with rind: (a) Picture taken optically, (b) SAFT processing at $22.25 \mathrm{GHz}$ focusing at the substrate or $74 \mathrm{~mm}$ from measurement plane, (c) Microwave holography focusing at $34 \mathrm{~mm}$, (d) Microwave holography focusing at substrate 


\section{Foam with Rind}

Now that it has been demonstrated that these methods can detect known voids in foam, this experiment shows that these imaging methods can be performed on an unknown specimen and much information about the SOFI can be revealed. A picture of the SOFI sample with rind is shown in Figure 3a. Note that the boot print on the upper left corner caused no internal damage to the SOFI and can be neglected. Figure $3 \mathrm{~b}$ shows the result of SAFT processing, and knit lines can be seen in this image. Microwave holography shows the knit lines between successive layers of foam spray in Figure 3c, and also clearly shows the exposed rind that appears on the lower right hand quadrant of the SOFI. Figure 3c also shows the two very small defects at the approximate $(x, y)$ coordinates of $(310 \mathrm{~mm}, 110 \mathrm{~mm})$ and $(540 \mathrm{~mm}, 410 \mathrm{~mm})$. The shadow of the first defect can be seen in Figure 3d when the holographic dataset was sliced at the depth corresponding to the location of the substrate. No other defects were discovered besides the reflection of the knit lines. The sample was not dissected for destructive examination since it is to be preserved for future evaluation and tests.

\section{SUMMARY}

It was shown that microwave imaging combined with SAFT and microwave holography can produce high resolution images of SOFI samples even with low power and low contrast of the specimen. Firstly, it was shown that the received signal power was sufficient by first showing that SOFI cubes could be found in air. Secondly, it was shown, from a more practical standpoint, that air voids in SOFI could be detected even under $140 \mathrm{~mm}$ (5.5 in) of SOFI. Lastly, the imaging algorithms were tested on an unknown SOFI sample where the knit lines close to the surface were shown and two small defects were successfully detected. The series of experiments performed demonstrate the capability of computational microwave imaging techniques to detect voids in SOFI. This investigation is ongoing at higher frequencies extending into the higher ends of millimeter wave spectrum. At these frequencies the size of a small antenna is a few millimeter across and when these algorithms are use for synthetically producing a larger antenna the resulting spatial resolutions will be on the order of a few $\mathrm{mm}$. Moreover, at these frequencies the available signal bandwidth is in tens of $\mathrm{GHz}$ which results in images with high range resolution. Therefore, at these frequencies 3D images of defects in SOFI can be produced that are as small as a few cubic millimeters.

\section{ACKNOWLEDGEMENT}

Funding for this work was provided by the NASA Marshall Space Flight Center through a Cooperative Agreement. The authors are also grateful to Mr. James. L. Walker for many insightful discussions on the nature of SOFI.

\section{REFERENCES}

1. Columbia Accident Investigation Board Report, NASA, August 2003.

2. S. Kharkovsky, F. Hepburn, J. Walker, R. Zoughi. Nondestructive Testing of the Space Shuttle External Tank Foam Insulation using Near-Field and Focused Millimeter Wave Techniques, Materials Evaluation, vol. 63, N5, 2005, pp.516-522.

3. S. Shrestha, S. Kharkovsky, R. Zoughi and F. Hepburn. Microwave and Millimeter Wave Nondestructive Testing of the Space Shuttle External Tank Insulating Foam, Materials Evaluation, vol. 63, N3, 2005, pp.339-344. 
4. Busse, L.J., "Three-Dimensional Imaging Using a Frequency-Domain Synthetic Aperture Focusing Technique," IEEE Transactions on Ultrasonics, Ferroelectrics, and Frequency Control, Vol. 39, No. 2, pp. 174-179, March 1992.

5. Sheen, D.M., D.L. McMakin, T.E. Hall, "Three-Dimensional Millimeter-Wave Imaging for Concealed Weapon Detection," IEEE Transactions on Microwave Theory and Techniques, Vol. 49, No. 9, September 2001.

6. Bois, K, L. Handjojo, A. Benally, K. Mubarak and R. Zoughi, "Dielectric Plug-Loaded Two-Port Transmission Line Measurement Technique for Dielectric Property Characterization of Granular and Liquid Materials," IEEE Transactions on Instrumentation and Measurement, vol. 48, no. 6, pp. 1141-1148, December 1999.

7. F.T. Ulaby, R.K. Moore and A.K. Fung, "Microwave Remote Sensing, Active and Passive" Vol. II, Artech House, Norwood, MA, 1986.

8. Yamani, Ahmed, "Three-Dimensional Imaging Using a New Synthetic Aperture Focusing Technique," IEEE Transactions on Ultrasonics, Ferroelectrics, and Frequency Control, Vol. 44, No. 4, July 1997.

9. Balanis, Constantine A., Antenna Theory: Analysis and Design, $2^{\text {nd }}$ Ed., John Wiley \& Sons, Inc., New York, 1997.

10. Bois, Karl, "Near-Field Microwave Inspection and Characterization of Cement Based Materials," Ph.D. Dissertation, Department of Electrical and Computer Engineering, Colorado State University, Fort Collins, CO 1999. 


\title{
MICROWAVE AND MILLIMETER WAVE IMAGING OF THE SPACE SHUTTLE EXTERNAL FUEL TANK SPRAY ON FOAM INSULATION (SOFI) USING SYNTHETIC APERTURE FOCUSING TECHNIQUES (SAFT)
}

\author{
J.T. Case', J. Robbins ${ }^{1}$, S. Kharkivskiy ${ }^{1}$, F. Hepburn ${ }^{2}$ and R. Zoughi ${ }^{1}$ \\ 'Applied Microwave Nondestructive Testing Laboratory (amnt), Electrical and Computer \\ Engineering Department, University of Missouri-Rolla, Rolla, MO 65409, USA \\ ${ }^{2}$ NASA George C. Marshall Space Flight Center, Huntsville, AL 35812, USA
}

\begin{abstract}
The Space Shuttle Columbia's catastrophic failure is thought to have been caused by a dislodged piece of external tank spray on foam insulation (SOFI) striking the left wing of the orbiter causing significant damage to some of the reinforced carbon/carbon leading edge wing panels. Microwave and millimeter wave nondestructive evaluation methods have shown great potential for inspecting SOFI for the purpose of detecting anomalies such as small air voids that may cause separation of the SOFI from the external tank during a launch. These methods are capable of producing relatively high-resolution images of the interior of SOFI particularly when advanced imaging algorithms are incorporated into the overall system. To this end, synthetic aperture focusing techniques (SAFT) are being developed. This paper presents some of the preliminary results of this investigation using SAFT-based methods and microwave holography at relatively low frequencies illustrating their potential capabilities for operation at millimeter wave frequencies.
\end{abstract}

Keywords: microwave holography, SAFT, SOFI

PACS: 42.30.Va, 42.40.Jv, 84.40.-x

\section{INTRODUCTION}

Spray on foam insulation (SOFI) is used to insulate the external fuselage of the Space Shuttle to maintain fuel, liquid hydrogen and liquid oxygen, at cryogenic temperatures [1]. During a launch, pieces of the SOFI may break off ranging in size from a few cubic millimeters to thousands of cubic centimeters. One such piece is thought to have damaged the reinforced carbon/carbon panels on the wing of the obiter Columbia [1]. The presence of an interior small void (or debond) in SOFI provides for higher internal pressure in the void during the high speed launch and may cause surrounding SOFI to break and dislodge from the tank. Therefore, a robust nondestructive testing and evaluation (NDT\&E) method is desired that is capable of detecting small voids in SOFI prior to a launch. 
Microwave and millimeter wave NDT\&E methods have shown great potential to produce relatively high-resolution images of voids inside SOFI test panels $[2,3]$. These methods are expected to be even more effective when combined with advanced imaging algorithms. Two such methods are presented in this paper, namely; synthetic aperture focusing technique (SAFT) [4] and microwave holography [5]. These methods are in many ways similar to one another. For processing purposes, it is assumed that the wave is not delayed as it propagates through SOFI. This is valid because SOFI has a permittivity of only 1.05 at X-band (8.2-12.4 $\mathrm{GHz})$ as compared to air with a relative permittivity of $1.00[3,6]$. Therefore, image processing is used to aid in finding weak reflections occurring at air and SOFI interfaces (i.e., void boundaries). The essence of each technique is to match the phase of the wave signal originating an arbitrary located target to measured points spaced regularly on a plane. For a signal originating at that target, processed signals add constructively and they add destructively for all surrounding points, consequently providing significant spatial discrimination resulting in a high-resolution image.

Synthetic aperture focusing technique (SAFT) can be used to synthetically focus a single frequency microwave image to a specific focal plane. For this method, a large, narrow beam antenna is synthesized by measuring with a small, broad-beam antenna at regular points on a grid, which results in images with high spatial resolution. The SAFT method described and used here was derived in the frequency domain and utilizes angular spectrum decomposition [4]. The original microwave image is decomposed onto a basis set of plane waves emitted from the center of the synthetic antenna array using the 2D FFT. The waves are then back-propagated to the focal plane and then to the measurement plane accounting for roundtrip back-propagation, and then they are composed back into normal space using the inverse 2D FFT. This yields a high resolution $2 \mathrm{D}$ image with lateral resolution of one-half the length of the dimension of the actual small microwave antenna and is independent of range or distance to the target [7]. To augment range resolution and image quality, it is possible to perform this algorithm at many frequencies within a waveguide band and average the intermediate data before the inverse 2D FFT is performed. However, this method may not produce high quality images for an object greater than the depth of focus [8].

Microwave holography, on the other hand, can be used to generate a full 3D representation of an object or embedded defect [5]. Thus, the 3D position and shape of the defect may be determined. This method utilizes a swept frequency measurement over the measurement plane resulting in a dataset of $(x, y, f)$. During image processing, spatial dimensions $(x, y)$ are transformed to spatial frequency dimensions $\left(k_{x}, k_{y}\right)$ by operating the 2D FFT independently on every measured frequency, $f$. Frequency $f$ can then be related to spatial frequency $k_{z}$ using the following dispersion relation [5]

$$
k_{x}^{2}+k_{y}^{2}+k_{z}^{2}=(2 k)^{2}
$$

such that

$$
k=\frac{2 \pi f}{c}
$$

where $c$ is the speed of light in vacuum. Real datasets must be re-sampled onto a regular spacing of $k_{z}$. The dataset can then be transformed to $(x, y, z)$ from $\left(k_{x}, k_{y}, k_{z}\right)$ using the inverse 3D FFT. Consequently, the resulting dataset is a volumetric representation of the defect and can 
be graphically illustrated as such. The spatial resolution is comparable to SAFT such that the range of the target is less than the dimension of the array. The range resolution associated with this holographic method is $c / 2 B$, where $B$ is the transmitted signal bandwidth [5]. For this paper, slices of the volume dataset are provided rather than a full three dimensional representation due to the nature of the samples used.

It is very important to note that this investigation uses relatively low frequencies that are not conducive for direct imaging of the SOFI $[2,3]$. However, as will be seen later, when using the image processing algorithms outlined above, reasonably high resolution images are obtained even at these relatively low frequencies. The ultimate goal of this investigation is to demonstrate the capability of these methods before they are applied to much higher frequencies like the millimeter wave frequency spectrum (e.g., 30-300 GHz).

\section{EXPERIMENTAL APPROACH}

All measurements were taken using an automated 2D scanner such that all measurement points were constrained to one plane. At each point, the microwave reflection coefficient at the aperture of an open-ended rectangular waveguide was measured. Three separate experiments were conducted for this investigation: inverse foam, void in foam, and foam with rind. The first experiment, inverse foam, utilizes SOFI in air, representing the inverse of a cube of void in SOFI. The inverse foam case was used because at the time there was no representative SOFI sample available. Thus, two SOFI cubes with side dimensions of $12.7 \mathrm{~mm}(0.5 \mathrm{in})$ were prepared and placed on an aluminum plate (substrate) $12.7 \mathrm{~mm}$ apart and $100 \mathrm{~mm}$ below the measurement plane. Measurements were taken at $\mathrm{Ku}$-band (12.4-18 GHz) at a sampling increment of $5 \mathrm{~mm}$ using an HP8510C Vector Network Analyzer. The second experiment, void in foam, was conducted on a $70 \mathrm{~mm}$ thick SOFI slab with $25 \mathrm{~mm}$ (1 in) diameter flat bottom holes drilled at the following depths: $25 \mathrm{~mm}$ (1 in), $19 \mathrm{~mm}$ (3/4 in), $13 \mathrm{~mm}(1 / 2 \mathrm{in}), 6 \mathrm{~mm}$ (1/4 in), and 3 (1/8 in). This slab was arranged in three different ways for three cases: 1$)$ backed by substrate, 2) backed by another $70 \mathrm{~mm}$ thick SOFI slab with no holes (blank sample) and substrate, and 3) covered by $70 \mathrm{~mm}$ thick blank SOFI and backed by substrate. In this way the locations of the holes within the overall SOFI thickness varied. Liftoff, the distance between the probe and the SOFI surface, was $10 \mathrm{~mm}$. Measurements were taken at K-Band (18-26.5 $\mathrm{GHz}$ ) at sampling increments of $2 \mathrm{~mm}$ using an Agilent 8361A PNA Series Vector Network Analyzer. The third case, foam with rind, consisted of a large $560 \mathrm{~mm} \times 560 \mathrm{~mm} \times 76 \mathrm{~mm}$ (22 in $\times 22$ in $\times 3$ in) SOFI sample with exposed rind backed by substrate. Measurements were taken at K-Band, $18-26.4 \mathrm{GHz}$, at a sampling increment of $2 \mathrm{~mm}$ and a liftoff of $10 \mathrm{~mm}$ using an Agilent 8361A PNA Series Vector Network Analyzer.

\section{RESULTS}

\section{Inverse Foam}

The first experiment analyzed is the inverse foam case of two SOFI cubes on top of a substrate. Using Friis transmission equation the reflection from the SOFI cubes was estimated to be $-70 \mathrm{~dB}$ in relation to the microwave power at the aperture of the waveguide. Using image theory the reflection from the substrate would be much greater at $-45 \mathrm{~dB}$ [9]. Although the relative signal strength from the SOFI cube is small, it is still above noise and within the measurement dynamic range of the network analyzer. The waveguide-to-air boundary at the opening of the waveguide aperture causes a large reflection and is the dominant contribution to the net reflection coefficient. However, contributions to the measured reflection coefficient by 
the waveguide-to-air boundary and the substrate can be coherently subtracted before image processing is implemented (i.e., calibration). This is done by subtracting the average reflection coefficient over the whole scan area, which is a reasonable estimation of both reflections since they are expected to remain constant over the scan area. Figure 1a shows the original image of the magnitude of reflection coefficient as measured by scanning the waveguide probe over the region of interest, which gives only a faint indication that something is present, and this is expected in a direct measurement before processing. Clearly, in this image there is no indication of two separate pieces of SOFI adjacent to one another. Figure $1 \mathrm{~b}$ shows the processed image using the SAFT algorithm described earlier. It is important to note that the contributions from the SOFI cubes, although small, can be resolved from the darker surroundings. It is also important to note that each SOFI cube can be resolved individually from the other even though the wavelength considered at $12.4 \mathrm{GHz}$ is $24 \mathrm{~mm}$ long compared to the size of the SOFI cubes, $12.7 \mathrm{~mm}$. This example clearly shows the effectiveness of this SAFT approach for obtaining high-resolution images. Figure 1c shows that microwave holography can further increase the quality of the image using the entire bandwidth available (12.4-18 $\mathrm{GHz}$ ). The quality is improved by further resolving the SOFI cubes and suppressing the background (or clutter) signal. This representative experiment demonstrates that the concept of using SAFT-based algorithms is not only practical, but also realizable despite the small waveguide probe power, small reflections from the SOFI, and comparatively large reflections from the substrate.

(a)

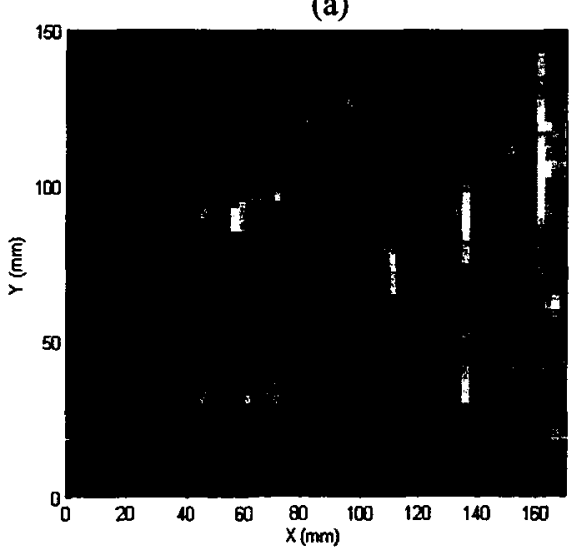

(b)

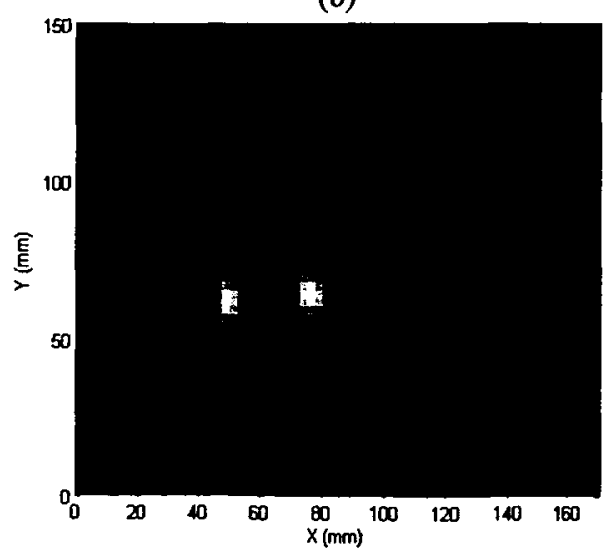

(c)

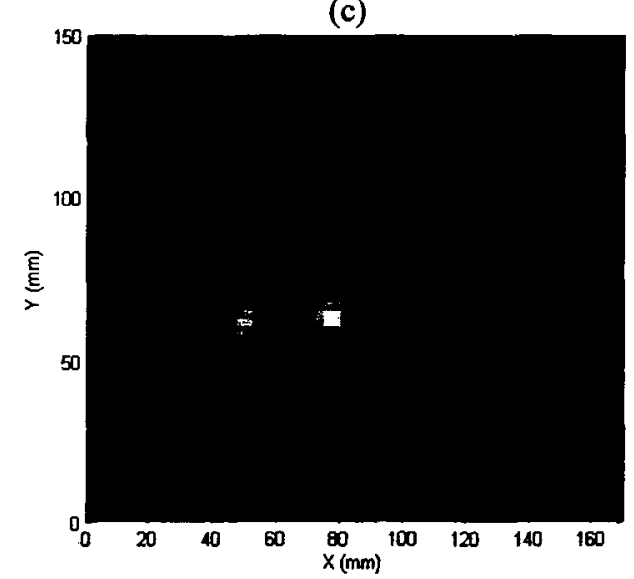

FIGURE 1. Inverse foam case: (a) original magnitude of reflection at $12.4 \mathrm{GHz}$, (b) SAFT processing at 12.4 $\mathrm{GHz}$ focusing at $105 \mathrm{~mm}$ from measurement plane, (c) microwave holography 
(a)

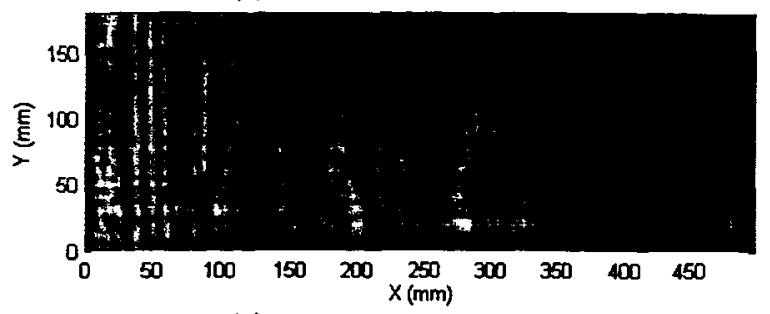

(c)

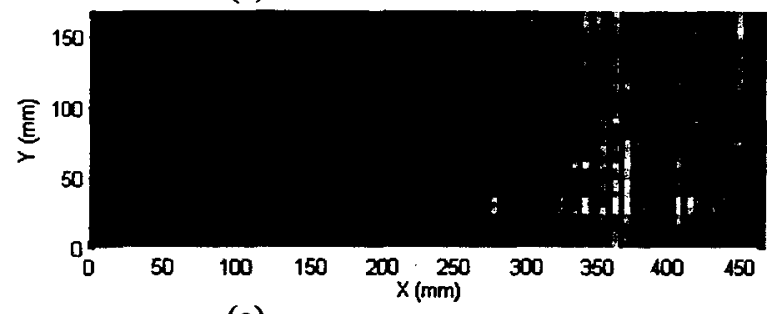

(e)

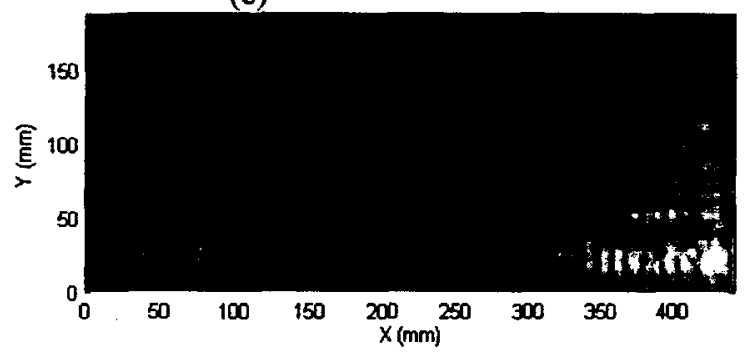

(b)

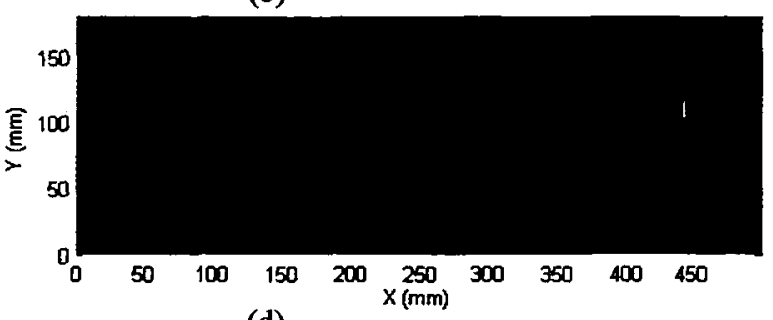

(d)

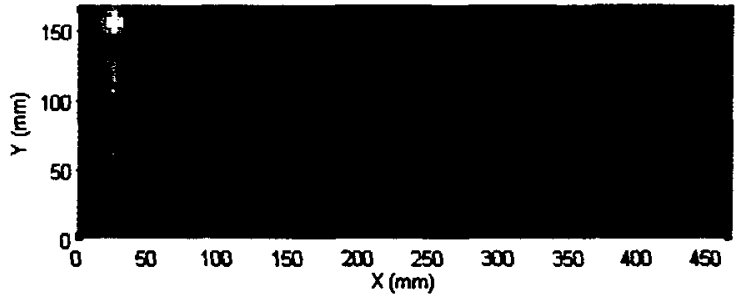

(f)

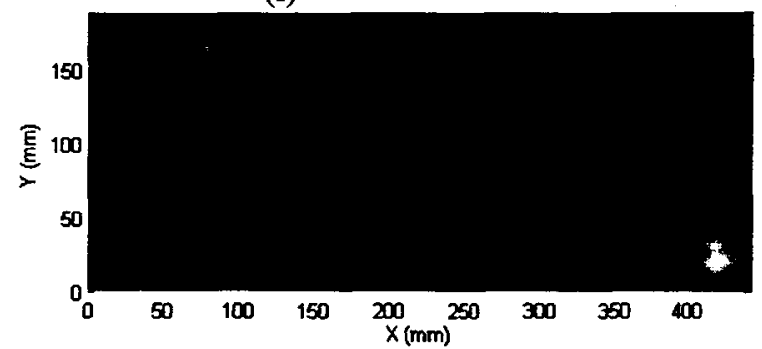

FIGURE 2. Void in foam: (a) case 1: SAFT processing at $22.25 \mathrm{GHz}$ focusing at $74 \mathrm{~mm}$ from measurement plane; (b) case 1: microwave holography slice at $74 \mathrm{~mm}(18-26.5 \mathrm{GHz})$; (c) case 2: SAFT processing at $22.25 \mathrm{GHz}$ focusing at 152 $\mathrm{mm}$; (d) case 2: microwave holography slice at $152 \mathrm{~mm}$; (e) case 3: SAFT processing at $22.25 \mathrm{GHz}$ focusing at $152 \mathrm{~mm}$; (f) case 3: microwave holography slice at $152 \mathrm{~mm}$.

\section{Void in Foam}

The first experiment demonstrated that foam could be detected in air. This experiment goes further to demonstrate the effectiveness of these algorithms for detecting voids in SOFI at different depths. This experiment was performed for three different cases as described in the experimental approach section. The first case consists of one SOFI slab with five flat bottom holes of different depths backed by an aluminum substrate. It is important to note the horizontal and vertical line aberrations occurring near the edges of the processed images. These are the result of two causes: the effects from the edges of the substrate and the rectangular, not square, nature of the dataset. Both can be minimized if the data set is cropped about a square region of interest, and future versions of the image processing code will minimize these aberrations. In Figure $2 \mathrm{a}$, SAFT processing shows four of the five holes starting at the deepest on the right and descending towards the left. Microwave holography in Figure $2 \mathrm{~b}$ also shows four holes. The fifth hole is not evident from Figure $2 b$ since it is nearly masked by the edge effects. In spite of this, the fifth hole can be seen as a persistent indication when viewing the adjacent focal planes at different depths (not shown here).

The second case is for two SOFI slabs such that the slab containing the holes is nearest to the measurement plane and is backed by another slab and the aluminum substrate. The best images were obtained by focusing on the shadows of the voids cast onto the substrate. As seen in Figure 2c, SAFT processing is inadequate for such a thick sample and random noise seems 
to overwhelm the results except for a hint of the dominant hole on the far right. It is thought that the reduction in the quality of the image is due to low signal strength and low range resolution. Range resolution is dependent on the bandwidth of the system, but SAFT is inherently single frequency and as such provides low range resolution. Although range resolution is not usually necessary for a quality $2 \mathrm{D}$ image, the features sought for at a specific focal plane are blurred out along the direction of range. Therefore, the voids are effectively masked by the noise/clutter. Microwave holography uses the full available frequency spectrum and provides better results, as shown in Figure 2d, which shows four of the five holes for this case.

The third case is of two SOFI slabs where the slab with holes was sandwiched between another slab and the aluminum substrate. Once again, SAFT algorithm performed poorly due to reasons already described, as shown Figure 2e. However, four of the five holes were successfully detected using microwave holography. The last and smallest hole is again dominated by edge effects, as shown in Figure $2 \mathrm{f}$. It is important to note that this third case demonstrates that voids can be seen through $140 \mathrm{~mm}$ (5.5 in) of SOFI using this microwave holography.

(a)

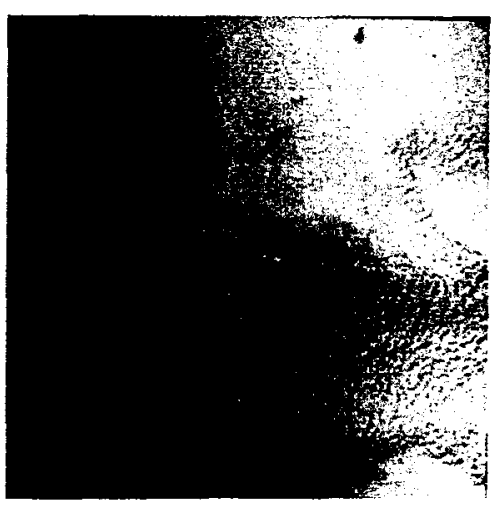

(c)

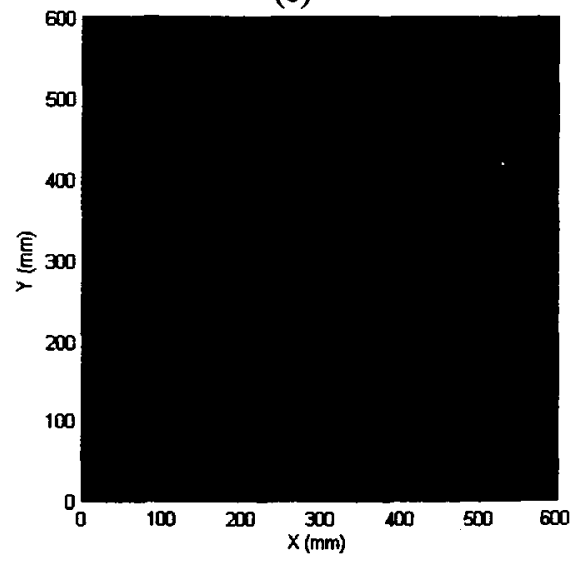

(b)

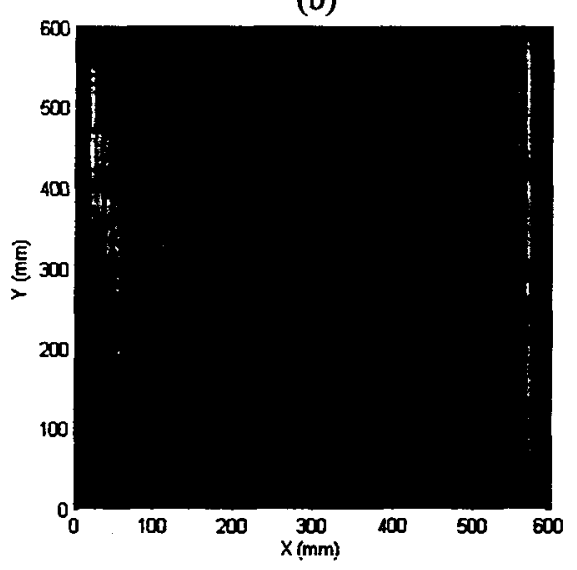

(d)

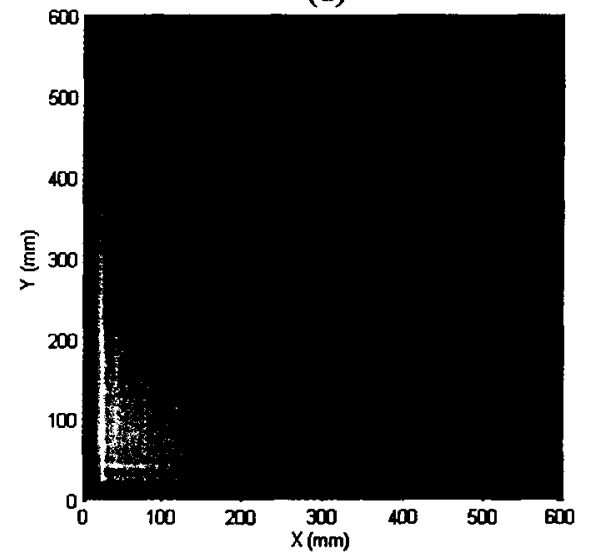

FIGURE 3. Foam with rind: (a) Picture taken optically, (b) SAFT processing at $22.25 \mathrm{GHz}$ focusing at the substrate or $74 \mathrm{~mm}$ from measurement plane, (c) Microwave holography focusing at $34 \mathrm{~mm}$, (d) Microwave holography focusing at substrate 


\section{Foam with Rind}

Now that it has been demonstrated that these methods can detect known voids in foam, this experiment shows that these imaging methods can be performed on an unknown specimen and much information about the SOFI can be revealed. A picture of the SOFI sample with rind is shown in Figure 3a. Note that the boot print on the upper left corner caused no internal damage to the SOFI and can be neglected. Figure $3 \mathrm{~b}$ shows the result of SAFT processing, and knit lines can be seen in this image. Microwave holography shows the knit lines between successive layers of foam spray in Figure $3 \mathrm{c}$, and also clearly shows the exposed rind that appears on the lower right hand quadrant of the SOFI. Figure $3 \mathrm{c}$ also shows the two very small defects at the approximate $(x, y)$ coordinates of $(310 \mathrm{~mm}, 110 \mathrm{~mm})$ and $(540 \mathrm{~mm}, 410 \mathrm{~mm})$. The shadow of the first defect can be seen in Figure $3 \mathrm{~d}$ when the holographic dataset was sliced at the depth corresponding to the location of the substrate. No other defects were discovered besides the reflection of the knit lines. The sample was not dissected for destructive examination since it is to be preserved for future evaluation and tests.

\section{SUMMARY}

It was shown that microwave imaging combined with SAFT and microwave holography can produce high resolution images of SOFI samples even with low power and low contrast of the specimen. Firstly, it was shown that the received signal power was sufficient by first showing that SOFI cubes could be found in air. Secondly, it was shown, from a more practical standpoint, that air voids in SOFI could be detected even under $140 \mathrm{~mm}$ (5.5 in) of SOFI. Lastly, the imaging algorithms were tested on an unknown SOFI sample where the knit lines close to the surface were shown and two small defects were successfully detected. The series of experiments performed demonstrate the capability of computational microwave imaging techniques to detect voids in SOFI. This investigation is ongoing at higher frequencies extending into the higher ends of millimeter wave spectrum. At these frequencies the size of a small antenna is a few millimeter across and when these algorithms are use for synthetically producing a larger antenna the resulting spatial resolutions will be on the order of a few mm. Moreover, at these frequencies the available signal bandwidth is in tens of $\mathrm{GHz}$ which results in images with high range resolution. Therefore, at these frequencies $3 \mathrm{D}$ images of defects in SOFI can be produced that are as small as a few cubic millimeters.

\section{ACKNOWLEDGEMENT}

Funding for this work was provided by the NASA Marshall Space Flight Center through a Cooperative Agreement. The authors are also grateful to Mr. James. L. Walker for many insightful discussions on the nature of SOFI.

\section{REFERENCES}

1. Columbia Accident Investigation Board Report, NASA, August 2003.

2. S. Kharkovsky, F. Hepburn, J. Walker, R. Zoughi. Nondestructive Testing of the Space Shuttle External Tank Foam Insulation using Near-Field and Focused Millimeter Wave Techniques, Materials Evaluation, vol. 63, N5, 2005, pp.516-522.

3. S. Shrestha, S. Kharkovsky, R. Zoughi and F. Hepburn. Microwave and Millimeter Wave Nondestructive Testing of the Space Shuttle External Tank Insulating Foam, Materials Evaluation, vol. 63, N3, 2005, pp.339-344. 
4. Busse, L.J., "Three-Dimensional Imaging Using a Frequency-Domain Synthetic Aperture Focusing Technique," IEEE Transactions on Ultrasonics, Ferroelectrics, and Frequency Control, Vol. 39, No. 2, pp. 174-179, March 1992.

5. Sheen, D.M., D.L. McMakin, T.E. Hall, "Three-Dimensional Millimeter-Wave Imaging for Concealed Weapon Detection," IEEE Transactions on Microwave Theory and Techniques, Vol. 49, No. 9, September 2001.

6. Bois, K, L. Handjojo, A. Benally, K. Mubarak and R. Zoughi, "Dielectric Plug-Loaded Two-Port Transmission Line Measurement Technique for Dielectric Property Characterization of Granular and Liquid Materials," IEEE Transactions on Instrumentation and Measurement, vol. 48, no. 6, pp. 1141-1148, December 1999.

7. F.T. Ulaby, R.K. Moore and A.K. Fung, "Microwave Remote Sensing, Active and Passive" Vol. II, Artech House, Norwood, MA, 1986.

8. Yamani, Ahmed, "Three-Dimensional Imaging Using a New Synthetic Aperture Focusing Technique," IEEE Transactions on Ultrasonics, Ferroelectrics, and Frequency Control, Vol. 44, No. 4, July 1997.

9. Balanis, Constantine A., Antenna Theory: Analysis and Design, $2^{\text {nd }}$ Ed., John Wiley \& Sons, Inc., New York, 1997.

10. Bois, Karl, "Near-Field Microwave Inspection and Characterization of Cement Based Materials," Ph.D. Dissertation, Department of Electrical and Computer Engineering, Colorado State University, Fort Collins, CO 1999. 